\title{
Continuous Representations of Interval Orders by Means of Two Continuous Functions
}

\section{Gianni Bosi · Asier Estevan}

Received: date / Accepted: date

\begin{abstract}
In this paper, we provide a characterization of the existence of a representation of an interval order on a topological space in the general case by means of a pair of continuous functions, when neither the functions nor the topological space are required to satisfy any particular assumptions. Such characterization is based on a suitable continuity assumption of the binary relation, called weak continuity. In this way, we generalize all the previous results on the continuous representability of interval orders, and also of total preorders, as particular cases.
\end{abstract}

Gianni Bosi

University of Trieste

Trieste, Italy

gianni.bosi@deams.units.it

Asier Estevan

Universidad Pública de Navarra. Institute INAMAT

Pamplona, Spain

Corresponding author: asier.mugertza@unavarra.es

This is a post-peer-review, pre-copyedit version of an article published in Journal of Optimization Theory and Applications. The final authenticated version is available online at: https://doi.org/10.1007/s10957-020-01675-0 
Keywords Interval order · Continuous numerical representation · Weak continuity

Mathematics Subject Classification (2010) 91B06 -91B16

\section{Introduction}

It is well known that the interest of interval orders in economics, social sciences and mathematical disciplines in general is related to the fact that they are not necessarily transitive binary relations, which, under certain conditions, can be fully represented by means of pairs of real-valued functions.

It is interesting to look for conditions implying the existence of a continuous representation of an interval order on a topological space by means of a pair of real-valued functions. Necessary and sufficient conditions for the existence of a continuous representation of an interval order on a connected topological space were presented by Chateauneuf [1]. Fishburn [2] defined two total preorders, the so called traces, which are naturally associated to any interval order on a set. Characterizations of the existence of a continuous representation of an interval order on a topological space were presented by Bosi et al. [3, Corollary 4.3] and Bosi et al. [4, Theorem 1], when the traces are required to be continuous, so that the representing functions turn out to be continuous utility functions for the traces. Nevertheless, a condition of this kind is not necessary for the existence of a continuous representation (see, e.g., Bosi et al. [4, Example 1]).

Necessary conditions for the existence of a continuous representation of an 
interval order, and respectively a characterization of the existence of a continuous representation, when the support set is finite, were presented by Bosi et al. [5, Proposition 2 and Theorem 6].

In this paper, we provide a characterization in the general case of the existence of a continuous representation of an interval order on a topological space, i.e. when neither the representing functions nor the topological space are required to satisfy any particular assumptions. Such characterization is based on a suitable continuity assumption of the interval order, called weak continuity, which was introduced by Bosi [6]. In this way, we generalize all the previous results on the continuous representability of interval orders, and also of total preorders, as particular cases. For example, we show that any weakly continuous interval order on a second countable topological space is continuously representable. This result may be viewed as a slight generalization of the famous continuous utility representation theorem of Debreu [7], according to which a total preorder on a second countable topological space admits a continuous utility representation. In the last section we show that our considerations can be easily generalized to the case of biorders (see Doignon et al. $[8])$.

\section{Notation and Preliminary Results}

An interval order $\precsim$ on an arbitrary nonempty set $X$ is a binary relation on $X$ which is reflexive and in addition verifies the following condition for all 
$x, y, z, w \in X$

$$
(x \precsim z) \text { and }(y \precsim w) \Rightarrow(x \precsim w) \text { or }(y \precsim z) \text {. }
$$

An interval order $\precsim$ on a set $X$ is total (i.e., for all $x, y \in X$ we have that either $x \precsim y$ or $y \precsim x$ ) and not necessarily transitive (see, e.g., Oloriz et al. [9]). The irreflexive part of an interval order $\precsim$ will be denoted by $\prec$ (i.e., for all $x, y \in X, x \prec y$ if and only if $(x \precsim y)$ and $\operatorname{not}(y \precsim x))$.

Fishburn [2] proved that if $\precsim$ is an interval order on a set $X$, then the following two binary relations $\precsim^{*}$ and $\precsim^{* *}$ on $X$ are both total preorders:

$$
\begin{gathered}
x \precsim^{*} y \Leftrightarrow(z \precsim x \Rightarrow z \precsim y) \text { for all } z \in X, \\
x \precsim^{* *} y \Leftrightarrow(y \precsim z \Rightarrow x \precsim z) \text { for all } z \in X .
\end{gathered}
$$

Obviously, if $\precsim$ is a total preorder $\precsim$ (i.e., $\precsim$ is reflexive, transitive and total), then $\precsim$ is an interval order. In this case, we have that $\precsim=\precsim^{*}=\precsim^{* *}$. The irreflexive parts of $\precsim^{*}$ and $\precsim^{* *}$ will be denoted by $\prec^{*}$ and $\prec^{* *}$.

If $R$ is a binary relation on a set $X$, then denote by $L_{R}(x)\left(U_{R}(x)\right)$ the lower (upper) section of any element $x \in X$ (i.e., for every $x \in X, L_{R}(x)=$ $\{y \in X: y R x\}$ and $\left.U_{R}(x)=\{y \in X: x R y\}\right)$. If $(X, R)$ is any related set, then a subset $D$ of $X$ is said to be $R$-decreasing if $L_{R}(x) \subset D$ for every $x \in D$.

We recall that a real-valued function $u$ on a preordered $\operatorname{set}(X, \precsim)$ is said to be increasing if, for all $x, y \in X$,

$$
x \precsim y \Rightarrow u(x) \leq u(y) .
$$

Further, a real-valued function $u$ on $X$ is said to be a utility function for a 
total preorder $\precsim$ on a set $X$ if, for all $x, y \in X$,

$$
x \precsim y \Leftrightarrow u(x) \leq u(y)
$$

A pair $(u, v)$ of real-valued functions on $X$ is said to represent an interval order $\precsim$ on $X$ if, for all $x, y \in X$,

$$
x \precsim y \Leftrightarrow u(x) \leq v(y) .
$$

We say that a pair $(u, v)$ of real-valued functions on $X$ almost represents an interval order $\precsim$ on $X$ if, for all $z, w \in X$,

$$
(z \precsim w \Rightarrow u(z) \leq v(w)) \text { and }(z \prec w \Rightarrow v(z) \leq u(w)) \text {. }
$$

It is clear that any representation $(u, v)$ of an interval order $\precsim$ on a set $X$ is also an almost representation.

An interval order $\precsim$ on a topological space $(X, \tau)$ is said to be upper (lower) semicontinuous if $L_{\prec}(x)\left(U_{\prec}(x)\right)$ is an open subset of $X$ for every $x \in X$. If $\precsim$ is both upper and lower semicontinuous, then it is said to be continuous .

Let us now recall the definition of weak continuity of an interval order on a topological space, which was introduced by Bosi [6, Definition 2.1] (see also Bosi and Zuanon [10]).

Definition 2.1 An interval order $\precsim$ on a topological space $(X, \tau)$ is weakly continuous if for every $x, y \in X$ such that $x \prec y$ there exists a pair $\left(u_{x y}, v_{x y}\right)$ of continuous real-valued functions on $(X, \tau)$ satisfying the following conditions: (i) $\left(u_{x y}, v_{x y}\right)$ almost represents $\precsim$; 
(ii) $v_{x y}(x)<u_{x y}(y)$

The concept of weak continuity described in Definition 2.1 is reminiscent of the concept of weak continuity of a preorder on a topological space. From Herden and Pallack [11, Definition 2.3], a (not necessarily total) preorder $\precsim$ on a topological space $(X, \tau)$ is said to be weakly continuous (see also Bosi and Herden [12]) if, for every $x, y \in X$ such that $x \prec y$, there exists a continuous increasing real-valued function $u_{x y}$ on $(X, \tau)$ such that $u_{x y}(x)<u_{x y}(y)$.

It is clear that if there exists a pair $(u, v)$ of continuous real-valued functions representing an interval order $\precsim$ on $(X, \tau)$, then $\precsim$ is weakly continuous.

Definition 2.2 We say that a total preorder $\precsim$ on a topological space $(X, \tau)$ is almost upper semicontinuous (almost lower semicontinuous) if there exists a mapping $L_{\prec}^{0}: X \rightarrow \tau$ such that, for every $x \in X, x \notin L_{\prec}^{0}(x), L_{\prec}^{0}(x) \supset L_{\prec}(x)$ and $L_{\prec}^{0}(x)$ is $\prec$-decreasing (respectively, there exists a mapping $U_{\prec}^{0}: X \rightarrow \tau$ such that, for every $x \in X, x \notin U_{\prec}^{0}(x), U_{\prec}^{0}(x) \supset U_{\prec}(x)$ and $U_{\prec}^{0}(x)$ is $\prec$-increasing).

It is clear that an upper (lower) semicontinuous total preorder on a topological space $(X, \tau)$ is almost upper (lower) semicontinuous.

Lemma 2.1 Let $\precsim$ be an interval order on a topological space $(X, \tau)$. If $\precsim$ is weakly continuous, then the following conditions hold:

(i) $\precsim$ is continuous;

(ii) ${ }^{*}$ is almost lower semicontinuous;

(iii) $\precsim^{* *}$ is almost upper semicontinuous. 
Proof Let $\precsim$ be a weakly continuous interval order on a topological space $(X, \tau)$.

(i). In order to prove that $\precsim$ is continuous, we first show that $L_{\prec}(x)=$ $\{z \in X: z \prec x\}$ is an open subset of $X$ for every $x \in X$. Consider some fixed $x \in X$, and let $z$ be any element of $X$ such that $z \prec x$. Since $\precsim$ is weakly continuous, there exists an almost representation $\left(u_{x z}, v_{x z}\right)$ of $\precsim$ such that $v_{x z}(z)<u_{x z}(x)$. Therefore, $v_{x z}^{-1}(]-\infty, u_{x z}(x)[)$ is an open subset of $X$ containing $z$ and contained in $L_{\prec}(x)$. Indeed, $x \precsim u$ implies that $u_{x z}(x) \leq v_{x z}(u)$ for every $u \in X$. Analogously it can be shown that $U_{\prec}(x)=\{z \in X: x \prec z\}$ is an open subset of $X$ for every $x \in X$.

(iii). Now let us show that $\precsim^{* *}$ is almost upper semicontinuous. Analogously it can be shown that $\precsim^{*}$ is almost lower semicontinuous. Define, for every $x \in X$,

$$
L_{\prec * *}^{0}(x)=\bigcup_{(u, v) \in U V_{\precsim}^{a c}} u^{-1}(]-\infty, u(x)[),
$$

where $U V_{\precsim}^{a c}$ stands for the set of all continuous almost representations of $\precsim$. It is immediate to check that $x \notin L_{\prec * *}^{0}(x)$, because otherwise there exists a pair $(u, v) \in U V_{\precsim}^{a c}$ such that $u(x)<u(x)$. It is easy to show that, for every $x \in X, L_{\prec * *}^{0}(x) \supset L \prec^{* *}(x)$. Indeed, $z \prec^{* *} x$ implies the existence of some point $\xi \in X$ such that $z \precsim \xi \prec x$, so that, from weak continuity of $\precsim$, there exists $\left(u_{\xi x}, v_{\xi x}\right) \in U V_{\precsim}^{a c}$ with $u_{\xi x}(z) \leq v_{\xi x}(\xi)<u_{\xi x}(x)$, implying $z \in L_{\prec * *}^{0}(x)$. Finally, since from the above considerations we have that $u(z) \leq u(x)$ for every $(u, v) \in U V_{\precsim}^{a c}$, and $z \prec^{* *} x$, the above defined set $L_{\prec * *}^{0}(x)$ is $\prec^{* *}$-decreasing. This consideration completes the proof. 
The following proposition holds, which reduces weak continuity of a total preorder (see Definition 2.1) precisely to the notion of continuity.

Proposition 2.1 Let $\precsim$ be a total preorder on a topological space $(X, \tau)$. Then the following conditions are equivalent:

(i) $\precsim$ is weakly continuous;

(ii) $\precsim$ is continuous.

Proof (i) $\Rightarrow$ (ii). See Lemma 2.1.

(ii) $\Rightarrow$ (i). Let $\precsim$ be a continuous total preorder on $(X, \tau)$. From Herden and Pallack [11, Lemma 2.2], for every pair $(x, y) \in X \times X$ such that $x \prec y$ there exists a real-valued continuous increasing function $u_{x y}$ such that $u_{x y}(x)<u_{x y}(y)$. Since it is immediate to check that the pair $\left(u_{x y}, u_{x y}\right)$ is a continuous almost representation of $\precsim$, we have that $\precsim$ is weakly continuous. This consideration completes the proof.

\section{Continuous Representability}

We recall the definition of a scale in a topological space and a lemma, the proof of which may be found for example in the proof of the lemma on pages 43-44 in Gillman and Jerison [13] (see also Burgess and Fitzpatrick [14]).

Definition 3.1 If $(X, \tau)$ is a topological space and $\mathbb{S}$ is a dense subset of $[0,1]$ such that $1 \in \mathbb{S}$, then a family $\left\{G_{r}\right\}_{r \in \mathbb{S}}$ of open subsets of $X$ is said to be a scale in $(X, \tau)$ if the following conditions hold:

(i) $G_{1}=X$; 
(ii) $\overline{G_{r_{1}}} \subseteq G_{r_{2}}$ for every $r_{1}, r_{2} \in \mathbb{S}$ such that $r_{1}<r_{2}$.

Lemma 3.1 If $\left\{G_{r}\right\}_{r \in \mathbb{S}}$ is a scale in a topological space $(X, \tau)$, then the formula

$$
u(x)=\inf \left\{r \in \mathbb{S}: x \in G_{r}\right\} \quad(x \in X)
$$

defines a continuous function on $(X, \tau)$ with values in $[0,1]$.

The following characterization of weak continuity of an interval order on a topological space was proven by Bosi and Zuanon [10, Proposition 3.2].

Proposition 3.1 Let $\precsim$ be an interval order on a topological space $(X, \tau)$. Then the following conditions are equivalent:

(i) $\precsim$ is weakly continuous;

(ii) For every pair $(x, y) \in X \times X$ such that $x \prec y$ there exist two scales $\left\{G_{r}^{*(x y)}\right\}_{r \in \mathbb{S}}$ and $\left\{G_{r}^{* *(x y)}\right\}_{r \in \mathbb{S}}$ in $(X, \tau)$ such that the family $\left\{\left(G_{r}^{*(x y)}, G_{r}^{* *(x y)}\right)\right\}_{r \in \mathbb{S}}$ satisfies the following conditions:

(a) $z \precsim w$ and $w \in G_{r}^{*(x y)}$ imply $z \in G_{r}^{* *(x y)}$ for every $z, w \in X$ and $r \in \mathbb{S}$

(b) $z \prec w$ and $w \in G_{r}^{* *(x y)}$ imply $z \in G_{r}^{*(x y)}$ for every $z, w \in X$ and $r \in \mathbb{S}$

(c) $x \in G_{r}^{*(x y)}$ and $y \notin G_{r}^{* *(x y)}$ for every $r \in \mathbb{S} \backslash\{1\}$.

We recall that an interval order $\precsim$ on a set $X$ is said to be $i . o$. separable if there exists a countable subset $D=\left\{d_{n}\right\}_{n \in \mathbb{N}}$ of $X$ such that for all $x, y \in X$ 
with $x \prec y$ there exist $d_{m}, d_{n} \in D$ such that $x \precsim^{*} d_{m} \prec d_{n} \precsim^{* *} y$ (see Bosi et al. [15]). In this case $D$ is said to be an i.o. order dense subset of the set $X$.

We now present a characterization of the existence of a continuous representation of an interval order on a topological space without imposing any restrictive condition.

Theorem 3.1 Let $\precsim$ be an interval order on a topological space $(X, \tau)$. Then the following conditions are equivalent:

(i) There exists a pair of continuous real-valued functions $(u, v)$ on $(X, \tau)$ representing $\precsim ;$

(ii) There exists a countable family $\left\{\left(u_{n}, v_{v}\right)\right\}_{n \in \mathbb{N} \backslash\{0\}}$ of pairs of continuous real-valued functions on $(X, \tau)$ with values in $[0,1]$ almost representing $\precsim$ such that for every $x, y \in X$ with $x \prec y$ there exists $n \in \mathbb{N} \backslash\{0\}$ with $v_{n}(x)<u_{n}(y)$;

(iii) $\precsim$ is i.o. separable and weakly continuous;

(iv) $\precsim$ is i.o. separable and for every pair $(x, y) \in X \times X$ such that $x \prec y$ there exist two scales $\left\{G_{r}^{*(x y)}\right\}_{r \in \mathbb{S}}$ and $\left\{G_{r}^{* *(x y)}\right\}_{r \in \mathbb{S}}$ in $(X, \tau)$ such that the family $\left\{\left(G_{r}^{*(x y)}, G_{r}^{* *(x y)}\right)\right\}_{r \in \mathbb{S}}$ satisfies the following conditions:

(a) $z \precsim w$ and $w \in G_{r}^{*(x y)}$ imply $z \in G_{r}^{* *(x y)}$ for every $z, w \in X$ and $r \in \mathbb{S}$;

(b) $z \prec w$ and $w \in G_{r}^{* *(x y)}$ imply $z \in G_{r}^{*(x y)}$ for every $z, w \in X$ and $r \in \mathbb{S}$;

(c) $x \in G_{r}^{*(x y)}$ and $y \notin G_{r}^{* *(x y)}$ for every $r \in \mathbb{S} \backslash\{1\}$;

Proof (i) $\Rightarrow$ (ii). Immediate, since there exists a strictly increasing and continuous function from the real line into $] 0,1[$. 
(ii) $\Rightarrow$ (i). It is straightforward to see that the pair of functions $(u, v)$ defined by $u=\sum_{1}^{\infty} \frac{u_{n}}{2^{n}}$ and $v=\sum_{1}^{\infty} \frac{v_{n}}{2^{n}}$ is a continuous representation of the interval order. (ii) $\Rightarrow$ (iii). Bosi and Zuanon [10, Proposition 2.1] actually proved that it is also the case that the above condition (ii) implies the existence of a continuous representation $(u, v)$ for the interval order $\precsim$. Therefore, condition (iii) immediately descends.

(iii) $\Rightarrow$ (ii). Since the interval order $\precsim$ is i.o.-separable, there exists a countable set $D \subseteq X$ such that for any $x \prec y$ there exist $d_{m}, d_{n} \in D$ such that $x \precsim^{*} d_{m} \prec d_{n} \precsim^{* *} y$. Since the interval order is weakly continuous, there exists a (possible uncountable) family $\left\{\left(u_{i}, v_{i}\right)\right\}_{i \in I}$ of continuous almost representations of $\precsim$ such that for any pair $x \prec y$ there is an index $i \in I$ such that $v_{i}(x)<u_{i}(y)$. Let us see that there also exists a countable family $\left\{\left(u_{j}, v_{j}\right)\right\}_{j \in J}$ of continuous almost representations of $\precsim$ that satisfies those conditions. We look for a countable set $\bar{D}$ that extends the initial i.o. order dense set $D$. For any $x \prec y$ there exist $d_{m}, d_{n} \in D$ such that $x \precsim^{*} d_{m} \prec d_{n} \precsim^{* *} y$, so -since $d_{m} \prec d_{n}$ - there exists a pair $\left(u_{m n}, v_{m n}\right)$ of continuous almost representations such that $v_{m n}\left(d_{m}\right)<u_{m n}\left(d_{n}\right)$. If it holds that $x \prec^{*} d_{m} \prec d_{n} \prec^{* *} y$, then $v_{m n}(x) \leq v_{m n}\left(d_{m}\right)<u_{m n}\left(d_{n}\right) \leq u_{m n}(y)$ is satisfied, so the strict inequality also holds true for the pair $x \prec y$. However, if $x \sim^{*} d_{m}$ or $y \sim^{* *} d_{n}$, the inequality $v_{m n}(x)<u_{m n}(y)$ may fail to be true. For the sake of convenience, define, for every $m, n \in \mathbb{N},{\overline{d_{m}}}^{*}=\left\{z \in X: z \sim^{*} d_{m}\right\}$, and ${\overline{d_{n}}}^{* *}=\left\{z \in X: z \sim^{* *} d_{n}\right\}$. In the case when $x \sim^{*} d_{m}$, we distinguish two cases.

(a) If there exists $d_{m}^{\prime} \in{\overline{d_{m}}}^{*}$ such that $v_{m n}\left(d_{m}^{\prime}\right)=\max \left\{v_{m n}(z): z \in\right.$ 
\left.${\overline{d_{m}}}^{*}\right\}$, then we shall add $d_{m}^{\prime}$ to the set $\bar{D}$ that extends $D$. Notice that, since $d_{m}^{\prime} \sim^{*} d_{m} \prec d_{n}$, then it holds $d_{m}^{\prime} \prec d_{n}$ too. Dually, if there exists $d_{n}^{\prime \prime} \in{\overline{d_{n}}}^{* *}$ such that $u_{m n}\left(d_{n}^{\prime \prime}\right)=\min \left\{u_{m n}(z): z \in{\overline{d_{n}}}^{* *}\right\}$, then we add $d_{n}^{\prime \prime}$ too.

(b) If there is no maximum (case (a)), then there is the supremum of the set $\left\{v_{m n}(z): z \in{\overline{d_{m}}}^{*}\right\}$. Hence, there exists a sequence $\left(d_{m n}^{k}\right)_{k \in \mathbb{N}} \subseteq{\overline{d_{m}}}^{*}$ such that the corresponding image $\left(v_{m n}\left(d_{m n}^{k}\right)\right)_{k \in \mathbb{N}}$ is strictly increasing (i.e. $\left.v_{m n}\left(d_{m n}^{1}\right)<v_{m n}\left(d_{m n}^{2}\right)<\cdots<v_{m n}\left(d_{m n}^{k}\right)<\cdots\right)$ and it converges to the supremum. In particular, there is an index $k_{0} \in \mathbb{N}$ such that $v_{m n}(x)<v_{m n}\left(d_{m n}^{k}\right)$ for every $k>k_{0}$. In that case, we add all these elements of the sequence $\left(d_{m n}^{k}\right)_{k \in \mathbb{N}} \subseteq{\overline{d_{m}}}^{*}$ (so, a countable number of elements) to $\bar{D}$. Again, notice that, since $d_{m n}^{k} \sim^{*} d_{m} \prec d_{n}$ (for any index $k$ ), then it holds $d_{m n}^{k} \prec d_{n}$ too, for every index $k$. We reason dually with the infimum in case there is no element $d_{n}^{\prime \prime} \in{\overline{d_{n}}}^{* *}$ such that $u_{m n}\left(d_{n}^{\prime \prime}\right)=\min \left\{u_{m n}(z): z \in{\overline{d_{n}}}^{* *}\right\}$ (case (a)). In order to summarize the previous arguments, define, for every pair $(m, n) \in \mathbb{N} \times \mathbb{N}$ such that $d_{m} \prec d_{n}\left(d_{m}, d_{n} \in D\right)$, for which, therefore, there exists an almost representation $\left(u_{m n}, v_{m n}\right)$ of $\precsim$ such that $v_{m n}\left(d_{m}\right)<u_{m n}\left(d_{n}\right)$, the following subsets $D_{m n}^{\prime}, D_{m n}^{\prime \prime}$ of $X$ :

$D_{m n}^{\prime}=\left\{\begin{array}{l}\left\{d_{m}^{\prime}\right\}: v_{m n}\left(d_{m}^{\prime}\right)=\max v_{m n}\left({\overline{d_{m}}}^{*}\right) \quad \text { if } \exists \max v_{m n}\left({\overline{d_{m}}}^{*}\right), \\ \left\{d_{m n}^{k}\right\}_{k \in \mathbb{N}}: \lim _{k \rightarrow+\infty} v_{m n}\left(d_{m n}^{k}\right)=\sup \left\{v_{m n}(z): z \in{\overline{d_{m}}}^{*}\right\} \quad \text { otherwise }\end{array}\right.$ $D_{m n}^{\prime \prime}=\left\{\begin{array}{l}\left\{d_{n}^{\prime \prime}\right\}: u_{m n}\left(d_{n}^{\prime \prime}\right)=\min u_{m n}\left({\overline{d_{n}}}^{* *}\right) \quad \text { if } \exists \min u_{m n}\left({\overline{d_{n}}}^{* *}\right), \\ \left\{d_{m n}^{k}\right\}_{k \in \mathbb{N}}: \lim _{k \rightarrow+\infty} u_{m n}\left(d_{m n}^{k}\right)=\inf \left\{u_{m n}(z): z \in{\overline{d_{n}}}^{* *}\right\} \quad \text { otherwise }\end{array}\right.$ 
Finally, define

$$
\bar{D}=D \cup \bigcup_{d_{m} \prec d_{n}, d_{m}, d_{n} \in D} D_{m n}^{\prime} \cup \bigcup_{d_{m} \prec d_{n}, d_{m}, d_{n} \in D} D_{m n}^{\prime \prime}
$$

in order to immediately realize that $\bar{D} \subseteq X$ is a countable set such that, for every pair $x \prec y$ (with $x, y \in X$ ), there exist $d_{m}, d_{n} \in \bar{D}$ such that $x \precsim^{*} d_{m} \prec d_{n} \precsim^{* *} y$, and at the same time there exists a pair of continuous almost representations $\left(u_{m n}, v_{m n}\right)$ such that $v_{m n}\left(d_{m}\right)<u_{m n}\left(d_{n}\right)$ as well as $v_{m n}(x) \leq v_{m n}\left(d_{m}\right)<u_{m n}\left(d_{n}\right) \leq u_{m n}(y)$. Thus we just need those pair of functions $\left(u_{i}, v_{i}\right)$ that distinguish the elements of $\bar{D}$, i.e. a countable number of functions $\left\{\left(u_{m n}, v_{m n}\right)\right\}_{m, n \in \mathbb{N}}$.

(iii) $\Rightarrow$ (iv). See Bosi and Zuanon [10, Proposition 3.2]

(iv) $\Rightarrow$ (ii). Based on Lemma 3.1 and Proposition 3.1, it is now nearly immediate to establish this implication.

Corollary 3.1 Let $\precsim$ be a weakly continuous interval order on a second countable topological space $(X, \tau)$. Then there exists a pair of continuous real-valued functions $(u, v)$ on $(X, \tau)$ representing .

Proof From Bridges [16, Proposition 2.3] and Lemma 2.1, (i), there exists a representation $\left(u^{\prime}, v^{\prime}\right)$ of the interval order $\precsim$, so that Theorem 3.1 applies.

It is now easy to check that Corollary 3.1 generalizes Debreu theorem.

Corollary 3.2 (Debreu theorem) Let $\precsim$ be a continuous total preorder on a second countable topological space $(X, \tau)$. Then there exists a continuous utility function $u$ on $(X, \tau)$ representing . 
Proof From Proposition 2.1, we have that $\precsim$ is weakly continuous, so that it is continuously representable by a pair $(u, v)$ of real-valued functions by Corollary 3.1. However, from the proof of Proposition 2.1, it is clear that we can consider $u_{n}=v_{n}$ for every $n \in \mathbb{N}^{+}$in condition (ii) of Theorem 3.1, so that actually we can take $u=v$, a continuous utility representation for $\precsim$.

Finally let's see that i.o.-separability is not a necessary condition for the existence of a continuous almost representation or for weak continuity (of course, without it a continuous representation cannot exist).

Example 3.1 Let $\precsim$ be the lexicographic total order defined on $X=] 0,1[\times] 0,1[$ :

$$
(a, b) \prec(x, y) \Longleftrightarrow\left\{\begin{array}{l}
a<x, \quad \forall b, y, \\
a=x, b<y .
\end{array}\right.
$$

So, $(a, b) \sim(x, y)$, if and only if $a=x$ and $b=y$. We endow $X$ with the order topology induced by the lexicographic order.

It is well-known (see Bridges and Mehta [17]) that the lexicographic total order on the plane fails to be perfectly-separable and, hence, it is not representable by means of a utility function $u$ such that $(a, b) \prec(x, y)$ if and only if $u((a, b)<u((x, y))$.

Let $\pi$ be the projection on the first coordinate, i.e. $\pi((x, y))=x$ for any $(x, y) \in X$. Now, for each $r \in] 0,1[$ we define the following function on $X$ :

$$
u_{r}((x, y))=\left\{\begin{array}{c}
0, \quad x<r, \\
y, \quad x=r, \\
1, \quad r<x .
\end{array}\right.
$$


Thus, functions $u_{r}$ (for every $\left.r \in\right] 0,1[$ ) and $\pi$ give rise to almost representations of the total order, since they satisfy that

1. $(a, b) \precsim(x, y) \Rightarrow v((a, b)) \leq v((x, y))$,

2. $(a, b) \prec(x, y) \Rightarrow v((a, b)) \leq v((x, y))$,

where $v=u_{r}$ or $v=\pi$. Moreover, it can be proved that $u_{r}$ is continuous (as well as projection $\pi$ ) with respect to the order topology induced by the lexicographic order. Hence, we conclude that the order is weakly continuous, since for any two points $(a, b)$ and $(x, y)$ with $(a, b) \prec(x, y)$ it holds that there exists a continuous almost representation $v$ satisfying that $v((a, b))<v((x, y))$ (in fact, $v=\pi$ if $a<x$ and $v=u_{a}$ if $a=x$ ).

\section{Continuous Representability of Biorders}

As a consequence of the study presented before for interval orders, we are also able to include some results concerning the continuous representability of biorders. First, we present some key definitions (see Doignon et al. [8]).

Definition 4.1 A binary relation $\prec$ from $A$ to $X$ is a biorder if it is Ferrers, that is, for every $a, b \in A$ and $x, y \in X$, $(a \prec x)$ and $(b \prec y)$ imply $(a \prec y)$ or $(b \prec x)$.

As usual, we write $x \precsim a$ whenever $\neg(a \prec x), a \in A, x \in X$.

Definition 4.2 A biorder $\prec$ from $A$ to $X$ is said to be representable (as well as realizable with respect to <; see Doignon et al. [8]), if there exists a pair of 
real-valued functions $v: A \rightarrow \mathbb{R}, u: X \rightarrow \mathbb{R}$ such that $a \prec x \Longleftrightarrow v(a)<u(x)$, $a \in A, x \in X$.

It is said to be representable with respect to $\leq$ (see [8]), if there exists a pair of real-valued functions $v: A \rightarrow \mathbb{R}, u: X \rightarrow \mathbb{R}$ such that $a \prec x \Longleftrightarrow v(a) \leq$ $u(x), a \in A, x \in X$.

Furthermore, we may extend the notion of almost representability used for interval orders to biorders in a natural manner:

Definition 4.3 We say that a pair $(v, u)$ of real-valued functions on $A$ and $X$, respectively, almost represents a biorder $\precsim$ from $A$ to $X$ if, for all $a \in A$ and $x \in X,(x \precsim a \Rightarrow u(x) \leq v(a))$ and $(a \prec x \Rightarrow v(a) \leq u(x))$.

Remark 4.1 Notice that the concept of almost representability for biorders is weaker than the representability with respect to $\leq$, as well as weaker than the representability with respect to $<$.

The following characterization is known in the literature (see Doignon [8] as well as Nakamura [18])

Theorem 4.1 $A$ biorder $\prec$ from $A$ to $X$ is representable (with respect to $<$ ) if and only if it is jointly dense, that is, there exists a countable set $D=A^{\prime} \cup X^{\prime}$ (with $A^{\prime} \subseteq A$ and $X^{\prime} \subseteq X$ ) such that for any pair $a \prec x$ there exist $b \in A^{\prime}$ and $y \in X^{\prime}$ such that $a \precsim * b \prec y \precsim * * x .^{1}$

Hence, now we may generalize the concept of weakly continuous representation for biorders.

\footnotetext{
${ }^{1}$ Here, as for interval orders, $\prec^{*}=\prec 0 \precsim$ and $\prec^{* *}=\precsim \circ \prec$.
} 
Definition 4.4 A biorder $\prec$ from a topological space $\left(A, \tau_{A}\right)$ to another one $\left(X, \tau_{X}\right)$ is weakly continuous if, for every $a \in A$ and $x \in X$ such that $a \prec x$, there exists a pair $\left(v_{a x}, u_{a x}\right)$ of continuous real-valued functions on $\left(A, \tau_{A}\right)$ and $(X, \tau)$ (respectively) satisfying the following conditions:

(i) $\left(v_{a x}, u_{a x}\right)$ almost represents $\prec$;

(ii) $v_{a x}(a)<u_{a x}(x)$.

Finally, we present as a corollary the following result, whose proof is reduced to a sketch since it is similar to that developed for interval orders.

Theorem 4.2 Let $\prec$ be a biorder from a topological space $\left(A, \tau_{A}\right)$ to $\left(X, \tau_{X}\right)$.

Then the following conditions are equivalent:

(i) There exists a pair of continuous real-valued functions $(v, u)$ on $\left(A, \tau_{A}\right)$ and $\left(X, \tau_{X}\right)$, respectively, representing $\prec$ with respect to $<$;

(ii) There exists a countable family $\left\{\left(v_{n}, u_{n}\right)\right\}_{n \in \mathbb{N} \backslash\{0\}}$ of pairs of continuous real-valued functions on $\left(A, \tau_{A}\right)$ and $\left(X, \tau_{X}\right)$ (respectively) with values in $[0,1]$ almost representing $\prec$ such that for every $a \in A$ and $x \in X$ with $a \prec x$ there exists $n \in \mathbb{N} \backslash\{0\}$ with $v_{n}(a)<u_{n}(x)$;

(iii) $\prec$ is jointly-dense and weakly continuous.

Proof (i) $\Rightarrow$ (ii). Immediate.

(ii) $\Rightarrow(\mathrm{i})$. As we did for interval orders, here again the pair of functions $(v, u)$ defined by $u=\sum_{1}^{\infty} \frac{u_{n}}{2^{n}}$ and $v=\sum_{1}^{\infty} \frac{v_{n}}{2^{n}}$ is a continuous representation of the biorder.

(ii) $\Rightarrow$ (iii). The weak continuity is trivial by (ii). Since (ii) implies (i), and 
this last one implies $\prec$ to be jointly dense, implication (ii) $\Rightarrow$ (iii) is clear.

(iii) $\Rightarrow$ (ii). Since the biorder is jointly-dense, there exists a countable set $D=A^{\prime} \cup X^{\prime}$ (with $A^{\prime} \subseteq A$ and $X^{\prime} \subseteq X$ ) such that for any pair $a \prec x$ there exist $b \in A^{\prime}$ and $y \in X^{\prime}$ such that $a \precsim^{*} b \prec y \precsim^{* *} x$. Since the biorder is weakly continuous, there exists a (possible uncountable) family $\left\{\left(v_{i}, u_{i}\right)\right\}_{i \in I}$ of continuous almost representations such that for any pair $a \prec x$ there is an index $i \in I$ such that $v_{i}(a)<u_{i}(x)$. The proof consists on finding a countable family $\left\{\left(v_{j}, u_{j}\right)\right\}_{j \in J}$ that satisfies those conditions. This is done as for the case of interval orders, extending the countable set $D=A^{\prime} \cup X^{\prime}$ to a bigger countable one $\overline{D^{\prime}}=\overline{A^{\prime}} \cup \overline{X^{\prime}}$, again distinguishing two cases depending on the existence of maximum and minimum values.

After the construction of that countable set $\overline{D^{\prime}}=\overline{A^{\prime}} \cup \overline{X^{\prime}}$, we only need those pair of functions $\left(v_{i}, u_{i}\right)$ that distinguish the elements of $\overline{A^{\prime}}$ from $\overline{X^{\prime}}$, i.e. a countable number of functions $\left\{\left(u_{m n}, v_{m n}\right)\right\}_{m, n \in \mathbb{N}}$.

\section{Conclusions}

In this paper, we have presented a characterization of the existence of a pair of continuous functions representing an interval order on a topological space. Such result does not utilize any restrictive assumption and it is based on a concept of continuity, namely weak continuity, which generalizes the classical continuity of total preorders. In this way, we have generalized all the previous results concerning both the existence of continuous representations of total preorders and the existence of continuous representations of interval orders. 
Acknowledgements Asier Estevan acknowledges financial support from the Ministry of Economy and Competitiveness of Spain under grants MTM2015-63608-P and ECO201565031. Gianni Bosi acknowledges financial support from the Istituto Nazionale di Alta Matematica "F. Severi" (Italy).

\section{References}

1. Chateauneuf, A.: Continuous representation of a preference relation on a connected topological space. Journal of Mathematical Economics 16, 139-146 (1987)

2. Fishburn, P.C., Interval Orders and Interval Graphs, Wiley, New York (1985)

3. Bosi, G., Candeal, J.C., Induráin, E.: Continuous representability of interval orders and biorders. Journal of Mathematical Psychology 51, 122-125 (2007)

4. Bosi, G., Campión, M.J., Candeal, J.C., Induráin, E.: Interval-valued representability of qualitative data: the continuous case. International Journal of Uncertainty, Fuzziness, and Knowledge-Based Systems 15, 299-319 (2007)

5. Bosi, G., Estevan, A.,Gutiérrez García, J., Induráin, E.: Continuous representability of interval orders: The topological compatibility setting. International Journal of Uncertainty Fuzziness and Knowledge-Based Systems 23, 345-365 (2015)

6. Bosi, G.: A note on continuity and continuous representability of interval orders, International Mathematical Forum 3, 1563-1568 (2008)

7. Debreu, G.: Continuity properties of Paretian utility. International Economic Review 5, $285-293(1964)$

8. Doignon, J.P., Ducamp, A., Falmagne, J.C.: On realizable biorders and the biorder dimension of a relation. Journal of Mathematical Psychology 28, 73-109 (1984)

9. Oloriz, E., Candeal, J.C., Induráin, E.: Representability of interval orders, Journal of Economic Theory 78, 219-227 (1998)

10. Bosi, G., Zuanon, M.: On the weak continuity of preference relations with nontransitive indifference: International Mathematical Forum 7, 849-853 (2012)

11. Herden, G., Pallack, A.: On the continuous analogue of the Szpilrajn Theorem I. Mathematical Social Sciences 43, 115-134 (2002) 
12. Bosi, G., Herden, G.: On a possible continuous analogue of the Szpilrajn theorem and its strengthening by Dushnik and Miller. Order 23, 271-296 (2006)

13. Gillman, L., Jerison, M.: Rings of continuous functions, Princeton, D. Van Nostrand Company (1960)

14. Burgess, D.C.J., Fitzpatrick, M.: On separation axioms for certain types of ordered topological space. Mathematical Proceedings of the Cambridge Philosophical Society $82,59-65(1977)$

15. Bosi, G., Candeal, J.C., Induráin, E., Oloriz, E., Zudaire, .M.: Numerical representations of interval orders. Order 18, 171-190 (2001)

16. Bridges, D.S.: Numerical representation of interval orders on a topological space. Journal of Economic Theory 38, 160-166 (1986)

17. Bridges, D.S., Mehta, G.B: Representations of Preference Orderings, Berlin-HeidelbergNew York: Springer-Verlag (1995)

18. Nakamura, Y.: Real interval representations. Journal of Mathematical Psychology 46, $140-177(2002)$ 